\title{
Distribución de Diplodia mutila FR. APUD Mont. En CATALUnya
}

\author{
Asunción MORET BENASET \& Martí NADAL PUIGDEFABREGAS
}

RESUMEN: Se ha recolectado Diplodia mutila en 62 localidades de las comarcas catalanas sobre 32 táxones de hospedantes, varios de los cuales no aparecen citados en la bibliografía; se indica también la fecha de recolección de cada muestra (día, mes y año).

Palabras clave: Diplodia, Coelomycetes.

RESUME: On récolte Diplodia mutila dans 62 localités des provinces catalanes sur 32 hôtes differents, dont plusieurs n'ont pas été cités dans la bibliographie; on signale aussi la date de récolte de chaque echantillon (jour, mois et an).

Mots clés:Diplodia, Coelomycetes.

\section{INTRODUCCIÓN}

Diplodia mutila no aparece citada en la bibliografía española hasta tiempos muy recientes (Nadal \& Moret, 1982), sin embargo parece tener por lo menos en Catalunya una distribución geográfica bastante amplia a la vez que es capaz de desarrollarse sobre un considerable número de especies vegetales entre las cuales se incluyen tanto especies espontáneas en el área biogeográfica considerada, como cultivadas para su aprovechamiento forestal y agro-alimentario.

Diplodia mutila Fr. apud Mont. es el estado anamórfico de Botryosphaeria stewensii Shoemaker. En dicho estado este hongo produce conidiomas inmersos en el sustrato, de tipo picnidio, los cuales son uniloculares y globulosos, de color castaño a negro y de pared gruesa; alcanzan hasta $600 \mu \mathrm{m}$ de diámetro y presentan un solo ostiolo central papilado. Los conidióforos que se forman a partir del estrato celular que tapiza el picnidio son hialinos, cilíndricos, de pared lisa, tabicados y a menudo ramificados en su base; pueden alcanzar una longitud de 4,5 $\mu \mathrm{m}$ y una anchura de 3 $5 \mu \mathrm{m}$. Las células conidiógenas son holoblásticas, integradas o discretas, de crecimiento limitado, cilíndricas, hialinas y lisas y forman un solo conidio apical. 
Los conidios, de pared gruesa y lisa, al principio, son unicelulares e hialinos y presentan una gútula central, pero al madurar toman gradualmente color castaño oscuro y se desarrolla un tabique transversal verdadero; su ápice es obtuso y la base truncada. Las dimensiones de los conidios maduros son 27-31 x 12-13,5 $\mu \mathrm{m}$.

Diplodia mutila presenta, como la mayoría de los hongos, una amplia distribución geográfica (Peace 1962 \& Sutton, 1980), de la que parece excluirse el continente americano; sin embargo, hay citas de islas próximas a Cuba. Las citas europeas corresponden a Inglaterra e Irlanda y, dentro del entorno mediterráneo, Francia y Chipre. Sin embargo, no hay ninguna cita en España hasta 1982 (Nadal \& Moret); 1984 (Moret \& Nadal) y 1985 (Nadal, Moret \& Monton). Con posterioridad a estas primeras citas se observó en una gama mucho más amplia de hospedantes que, a su vez, fueron recolectados en un área más extensa; no ha sido posible, no obstante, recolectar muestras infectadas en todos los municipios de las comarcas catalanas. Algunas comarcas quedaron fuera del programa inicial de prospección, pero en otras ocasiones los resultados negativos de los análisis de los municipios prospectados hace que éstas no aparezcan citadas. Por otra partre, algunos municipios citados se hallan en zonas prácticamente limítrofes con comarcas no prospectadas; cabe esperar, pues, que la prospección más exhaustiva permitirá en un futuro conocer mejor la distribución geográfica de esta especie en Catalunya.

Dado que Diplodia mutila es una especie nueva para la micoflora catalana y española, nos proponemos como objetivo del presente trabajo determinar las áreas biogeográficas de Catalunya ocupadas por este hongo así como la confección de una relación de hospedantes para compararla con la indicada en la bibliografía.

\section{MATERIAL Y MÉTODOS}

Se ha recolectado material vegetal infectado por Diplodia mutila en 22 de las 41 comarcas catalanas y en un total de 63 municipios.

Una parte considerable del material vegetal estudiado ha sido aportado por el Servei de Protecció dels Vegetals del Departament d'Agricultura Ramaderia i Pesca de la Generalitat de Catalunya.

Se ha analizado material vegetal fresco procedente de diversas comarcas catalanas tratándolo de dos formas distintas. En la mayoría de muestras, después de observar la sintomatología que presentaba la planta afectada, se localizaron los picnidios (tipo de frutificaciones de este hongo) con la ayuda de un microscopio estereoscópico; a partir de los picnidios se confeccionaron preparaciones microscópicas que se estudiaban posteriormente. En otras ocasiones se procedía al aislamiento del hongo en cultivo puro, utilizando como sustrato base PDA (Potato-Dextrosa-agar) y siguiendo para tal fin la técnica de Helmer.

\section{RESULTADOS}

Se indican las fechas y localidades de recolección de las especies hospedantes agrupadas en comarcas. 
ALT CAMP: Rodonya: Vitis vinifera L. 02-VI-86.

ALT EMPORDÁ: Garriguella: Vitis vinifera L. cv. carinyena, 10-IX-80 y 26-IV-85; Vitis vinifera L. cv. carnatxa, 10-IX-85; Vitis vinifera L. cv. lladosa, 26-IV-85; Vitis vinifera L., 04-V-87. Riumors: Ulmus minor Mill., 21-II-89. Sant Pere Pescador: Malus domestica Bork. var. golden, 05-III-86; Pyrus dulcis (Miller) D.A. Webb, 05-III-86. Torroella de Fluvia: Prunus amygdalus Batsch., 09-II-88; Prunus persica (L.) Batsch., 09-II-88.

ALT URGELL: Ribera d'Urgellet: Juglans regia L., 27-IX-87. La Seu d'Urgell Pyrus communis L., 06-VII-88.

BAIX CAMP: Alforja: Quercus ilex L., 12-VI-86. Almoster: Quercus ilex L., 23-II86. Reus: Prunus dulcis L., 24-III-87. Ruidecanyes: Quercus ilex L., 20-II-88.

BAIX EMPORDÁ: Bellcaire d'Empordá: Prunus persica (L.) Bar. var. nectarina, 24VI-88. La Bisbal d'Empordá: Platanus orientalis L., 03-X-88. Gualta: Acer platanoides L., 21-XI-88; Prunus persica (L.) Batsch., 19-IV-88.

BAIX LLOBREGAT: Begues: Quercus ilex L., 05-IX-83 y 12-IX-84. Castelldefels: Pinus pinea L., 19-II-85. Esparraguera: Populus nigra L., 21-X-85. Sta. Coloma de Cervello: Prunus domestica L., 13-III-87.

BAIX PENEDÉS: Banyeres del Penedés: Quercus ilex L., 20-VIII-85. St. Pau d'Ordal: Vitis vinifera $\mathrm{L} ., 21-\mathrm{V}-84$.

BAGES: Monistrol de Montserrat: Quercus ilex L., 15-III-80.

BERGUEDA: Gironella: Juniperus communis L., 04-V-87.

GIRONES: Fornells de la Selva: Cupressus sempervirens L., 30-VIII-88; Pyrus malus L. cv. early Blas., 04-IV-84; Pyrus malus L., 04-IV-84; Prunus avium L., 30-VIII-88.

MARESME: Cabrils: Quercus ilex L., 15-I-81. Vilassar de Mar: Rosa sp., 02-II-88. MONTSIA: St. Carles de la Rapita: Phoenix canariensis Chab., 04-II-88.

NOGUERA: Albesa: Pyrus communis v. gral. Leclerk., 10-XII-85; Prunus persica (L.) Batsch., 10-VIII-84. Artesa de Segre: Pyrus malus L., 26-VIII-88. Torrelameu: Pyrus communis L. var. devoe, 20-XI-86; Pyrus communis L., 20-XI-86.

OSONA: Centelles: Cedrus sp., 29-VI-88. Perafita: Fraxinus excelsior L., 27-VIII-86; Populus nigra L., 24-VII-85. Seva: Pinus nigra Arnold, 24-V-84.

PALLARS JUSSA: Tremp: Prunus dulcis (Miller) D.A Webb, 16-II-84.

PLA D’URGELL: Bell-Lloc d'Urgell: Pyrus communis L. cv. devoe, 29-V-86; Pyrus communis L., 29-V-86. Bellvis: Pyrus malus L., 20-VI-84; Prunus domestica L., 12-II-86. Golmes: Pyrus communis L., 02-IX-82. Miralcamp: Pyrus malus L., 10-VII-87. Sidamon: Pyrus communis L., 03-IV-87. Torregrossa: Pyrus malus L., 09-XII-83; Pyrus communis L., $29-X-87$

RIBERA D'EBRE: Ginestar: Prunus persica (L.) Batsch., 14-IV-87. Mora la Nova: Prunus persica (L.) Batsch., 14-IV-87.

SEGRIA: Alcarras: Pyrus communis L., 12-XII-82. Alcoletge: Pyrus malus L., 27-X86. Alguaire: Pyrus malus L., 23-XII-86. Lleida: Pyrus malus L., 21-V-84, 29-V-84 y 08-X87; Pyrus communis L., 15-VII-85, 15-X-87 y 18-III-88; Prunus persica (L.) Batsch., 10-III86. Puigverd de Lleida: Pyrus malus L., 30-I-86. Sudanell: Pyrus malus L., 12-VII-83. Torres de Segre: Pyrus communis L.cv. delbard., 25-II-85; Pyrus communis L., 25-II-85. Torre-Serona: Pyrus malus L., 02-X-87; Pyrus communis L., 02-X-87.

SELVA: Amer: Corylus avellana L., 08-V-87. Arbucies: Abies alba x A. pinsapo, 11-12-86; Malus domestica Borkh., 30-III-84. Caldes de Malavella: Cedrus sp., 30-III-87. Ruidellots de la Selva: Quercus ilex L., 12-VI-86. Sta. Coloma de Farners: Corylus avellana L. cv. negret, 
20-VI-85; Cupressus sempervirens L., 08-II-88; Pyrus communis L., 17-I-84.

TERRA ALTA: Gandesa: Pinus halepensis Mill., 06-VII-84; Vitis vinifera L., 02-X-86.

VALLES OCCIDENTAL: Caldes de Montbuy: Quercus ilex L., 08-IV-84 y 19-XII-88. Matadepera: Picea sp., 21-XI-86. Palau de Plegamans: Quercus ilex L., 18-III-84 y 19-XII84. St. Cugat del Vallés: Celtis australis L., 16-V-88 y Ulmus pumila L., 25-V-85.

VALLES ORIENTAL: Mollet del Valles: Pinus nigra Arnold., 06-XII-84. Vallgorguina: Quercus suber L., 09-VI-84. Vallromanes: Pinus halepensis Mill., 18-XII-86.

La distribución biogeográfica de D. mutila en Catalunya es muy amplia; se ha recolectado en 50 localidades distintas distribuidas entre las diversas comarcas.

Diplodia mutila presenta según la bibliografía una una gama de hospedantes relativamente amplia que comprende especies vegetales de grupos sistemáticos muy diversos, gimnospermas y angiospermas. Como característica común de los hospedantes, hay que destacar que la mayoría de ellos son de porte arbóreo arbustivo (leñosos); sin embargo Sutton, 1981, cita dos especies de porte herbáceo, Solanum lycopersicum y Solanum tuberosum (ambas de la familia Solanaceae) sobre las cuales nosotros no la hemos encontrado.

Hemos observado la especie objeto de estudio en 25 especies de hospedantes, todos ellos leñosos.

Relación de hospedantes: Abies alba Miller x Abies pinsapo Boiss.; Acer platanoides L.; Cedrus sp.; Celtis australis L.; Corylus avellana L.; Cupressus sempervirens L.; Fraxinus excelsior L.; Juglans regia L.; Juniperus communis L.; Pyrus malus L. Phoenix canariensis Chabaud.; Picea sp.; Pinus halepensis Mill. Pinus nigra Arnold.; Pinus pinea L.; Pyrus communis L.; Platanus orientalis L.; Populus nigra L.; Prunus dulcis (Miller) D.A. Webb.; Prunus avium L.; Prunus domestica L.; Prunus persica (L.) Batsch,; Prunus persica (L.) Batsch. cv. nectarina; Quercus ilex L.; Quercus suber L.; Rosa sp.; Ulmus pumila; Vitis vinifera L. cv. carinyena; Vitis vinifera L. cv. garnatxa; Vitis vinifera cv. lladosa; Vitis vinifera L.

De la relación antes indicada aparecen citados como hospederos en la bibliografía: Fraxinus excelsior L., Pyrus malus L., Pyrus comunis L., Prunus persica (L.) Batsch., Ulmus sp. y Vitis sp. asi como algunas especies exóticas del género Pinus ( $P$. caribaea y $P$. patula).

\section{BIBLIOGRAFÍA}

NADAL, M. \& A. MORET -1982- Espècies fitoparàsites de la Fam. Esferopsidàcies a Catalunya. Collectanea Botanica Vol. XIII. Barcelona.

MORET, A. \& M. NADAL -1984- Micosis de les Gimnospermes a Catalunya. X Jornadas de Productos Fitosanitarios. Barcelona.

NADAL, M., A. MORET \& C. MONTÓN -1985- Micosis d'algunes espècies del gènere Quercus. XI Jornadas de Productos Fitosanitarios. Barcelona.

PEACE, T.R. -1962- Pathology of Trees and Shrubs. Claredon Press Oxford.

SUTTON, B.C. -1980-The Coelomycetes. Commonwealth Mycological Institute. Kew, Surrey, England.

(Aceptado para su publicación en Junio de 1.990)

Dirección de los autores: Departamento de Biología Vegetal. Fitopatología. Facultad de Biología. Universitat de Barcelona. 